\title{
John-Arne Røttingen
}

Over 180 clinical trials of proposed COVID-19 drugs are already recruiting patients, and another 150 are registered to start recruiting patients soon. But many of these trials are small and not designed to identify the best treatment strategies for the COVID-19 pandemic. For Chief Executive of the Research Council of Norway John-Arne Røttingen, a more collaborative approach is now needed. And as Chair of the Executive Group and the International Steering Committee of the WHO's recently launched Solidarity trial, he hopes this mega-trial can provide a blueprint, he told Asher Mullard.

Already 70 countries have said they will join the Solidarity trial, paving the way to test the efficacy of the most promising agents at scale. For now, it is set up to test Gilead's RNA-polymerase inhibitor remdesivir, the antimalarials hydroxychloroquine and chloroquine, the HIV protease inhibitors lopinavir plus ritonavir, and lopinavir and ritonavir in combination with the immunomodulatory agent IFN $\beta_{1 \mathrm{a}}$. Results from the adaptive trial could be available within 12-16 weeks.

But vaccines are needed too. Røttingen has first-hand insight into the pain points ahead here, from his time overseeing an umbrella trial of Ebola vaccines and as founding CEO of the Coalition for Epidemic Preparedness Innovations (CEPI), a global alliance set up in 2016 to coordinate the development of new vaccines in times of crisis.

Nature Reviews Drug Discovery spoke with Røttingen about the Solidarity trial, the challenges ahead for vaccines and the global response to COVID-19.

\section{Q. What do you make of the more than} 180 trials of potential COVID-19 drugs that are already ongoing?

It's encouraging in the sense that it is really important to do trials in this situation, so that we can actually get solid evidence instead of just operating blind. Randomized trials are really important. The challenge though is the scale of these trials is too small, and the variation in terms of how they are being run is too large. They aren't really designed to answer the questions that need to be answered.

Q How will the Solidarity trial address this? Solidarity provides us with a great way of achieving fast-track data generation for promising candidates, on the most important clinical outcomes, in a very simple, large-scale mega-trial.
What we've coined is this novel sort of a mother-daughter relationship between trials, where a core protocol can be implemented at global scale, while countries with more capacity can collect much more detailed clinical trial data. And everyone will be using the same randomization rules, clinical end points, standard of care and inclusion-exclusion criteria.

\section{Q How big will the trial be, and what effect size is it powered to detect?}

We have deliberately not done power calculations, and are rather relying on the adaptive design and the interim analysis. So, the size will depend on the clinical effect of the drugs we are testing. The bigger the effects, the fewer patients we will need and the faster we can stop.

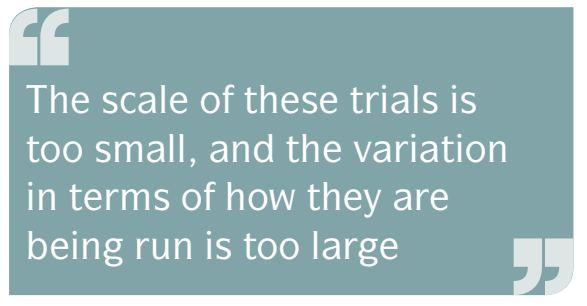

Q The WHO has disclosed the first four arms for this adaptive trial, but has said it might add more arms. How will this work?

We have appointed an independent data and safety monitoring committee, and they will be the only ones who can do interim analyses, look at the data and advise in terms of stopping arms as well as stopping the trial overall. Then, of course, there is the question of adding new arms as we go. The challenge is that if we add more arms, it will take longer to collect solid data on the therapeutic options that are in the existing arms. So that will be an issue that the data committee needs to look into. And this will depend in part on recruitment rates: if we can really speed up recruitment - and that's why we want to do this at scale in many countries - there will definitely be opportunities to increase the number of arms in the trial.

Another option could be that once we have enough data, we will conclude this first Solidarity trial and then restart another with a new set of arms, including potentially any drugs that have demonstrated an effect as standard of care. But this decision will be driven by the data.

Smaller trials can still be hypothesisgenerating, in that they will give us indications as to promising drugs to look at. But then these drugs will still need to be tested in larger trials, and Solidarity could be a mechanism to do that.

\section{Q This first trial is looking at hospitalized} patients with COVID-19. Why start there? That decision was made based on what is easiest to do currently, and we know that the testing capacity available in many countries right now means that only patients with severe symptoms are tested fully. And so it's easiest to have the inclusion at the hospital level. Of course, our trial is designed so that we will collect data on disease severity so that we can do subgroup analyses in the future.

can really speed up recruitment - and
that's why we want to do this at scale in
many countries - there will definitely be
opportunities to increase the number of
arms in the trial.
Another option could be that once we
have enough data, we will conclude this first
Solidarity trial and then restart another with
a new set of arms, including potentially any
drugs that have demonstrated an effect as
standard of care. But this decision will be
driven by the data.
Smaller trials can still be hypothesis-
generating, in that they will give us
indications as to promising drugs to look at.
But then these drugs will still need to be
tested in larger trials, and Solidarity could
be a mechanism to do that.
Q This first trial is looking at hospitalized
Tatients with COVID-19. Why start there?
That decision was made based on what is
easiest to do currently, and we know that the
igting capacity available in many countries
ight now means that only patients with
evere symptoms are tested fully. And so it's
asiest to have the inclusion at the hospital
vel. Of course, our trial is designed so
future.

Q. Antiviral agents may provide the most benefit when they are used earlier in the course of disease. Is there scope for a future master protocol trial looking at earlier intervention?

I believe that we might need that as well. In terms of prevention, there is already a planned large prevention trial with chloroquine or hydroxychloroquine. So that's one option. The other option is to start a trial in patients with mild symptoms. But the ability to get that running depends on better testing. 


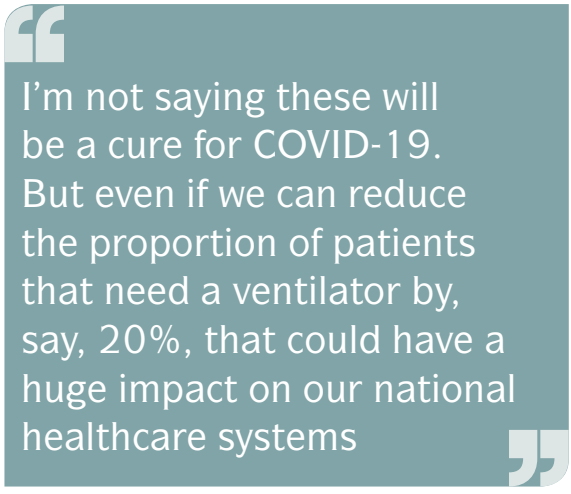

Q. Do you hope that Solidarity will provide baseline standards of care, end points, inclusionexclusion criteria and case report forms for others who are running standalone trials? I hope this will happen. Solidarity sets out clinical outcomes, inclusion-exclusion criteria, and a baseline standard of care. The daughter trials also establish even more detailed case report forms, which would be particularly useful for some of the newer drugs in development.

\section{Q What are realistic expectations for the efficacy of these first-round drugs?}

I don't have specific numbers in mind. And I've tried to be very clear on why we're doing this. I don't want to set expectations too high, but I do want to communicate that these are potentially promising therapeutics. The way I see it is that instead of just using drugs through sort of experimental use or compassionate use, we need to have evidence on whether or not they work. This is a way to give patients the best available treatments, but under a mechanism where we can actually learn from it.

I'm not saying these will be a cure for COVID-19. But even if we can reduce the proportion of patients that need a ventilator by, say, $20 \%$, that could have a huge impact on our national healthcare systems, and really improve the situation a lot. But it will do nothing to overall transmission rates and infection numbers.

Q Which brings us to the need for vaccines. Yes. I believe that a vaccine is a key long-term solution. I think it's really important to have high-speed vaccine development. I would like to see - after we have solid phase I trials on vaccine candidates - a parallel clinical development track for these.

\section{Q What are the key considerations on vaccines, beyond efficacy?}

One of the big challenges for vaccines is safety, given how widely these would be used. This is especially true for completely new vaccine platforms, such as mRNA and DNA vaccines that have yet to make it to market.

For clinical trials, there will need to be key decisions around which patient populations we would do the trials in. We will need a population with sufficiently high risks of actually getting COVID-19, so that there are enough events in the trials, but at the same time we do not want to do it in communities where there are already high levels of immunity. Similarly, we will want to see whether these vaccines work in elderly individuals and people with comorbidities, but the challenge with these elderly populations is that they typically have weaker immune responses. So there needs to be some consideration of the trade-offs in terms of the types of people you would want to test the vaccines in.

Another big challenge for vaccines compared with therapeutics would be scale. So volume of manufacturing will be a key challenge.

Then we have the access issues. What mechanisms could ensure fair distribution of vaccines? These vaccines are now being developed with public or philanthropic funding. So we really need to establish clear systems of fair access, where purchasing power and geographic production should not be the limiting factors.

\section{Q Is there enough funding in place for all this?}

It's still too limited. The calculations that have been done indicate that we ideally should have collectively US $\$ 2$ billion for vaccine development and testing. We have not been able to mobilize all that yet. But some countries have really contributed.
It's interesting that we've seen that the IMF, World Bank and sovereign banks really can mobilize resources for COVID-19. But these mechanisms provide funding to sovereign states, and we haven't established clear mechanisms for global pooled funding for technology development. This is something we need to think about, both for COVID-19 and for future situations.

Q You and your colleagues proposed the need for the WHO's R\&D Blueprint, following the 2014 Ebola outbreak, recognizing that we should start preparing for future infectious disease crises. Presumably, you hoped then that we'd have more time to prepare. What does the response to COVID-19 say about how well prepared we were? We were not prepared sufficiently. We have been talking about a pandemic forever. Of course, first and foremost we expected a flu pandemic, and I think we would have been better prepared if we had the flu pandemic. But we were not prepared for this. Not even the scientists that were closest to this sort of prediction were prepared.

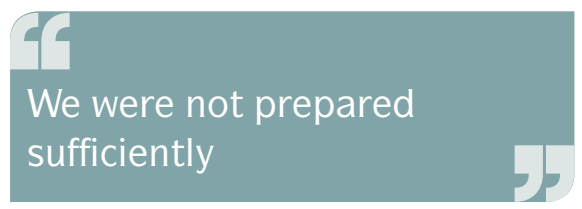

That said, I think we are now in a good position of international collaboration. And I think we are in a better position now than we were with Ebola.

Of course, Ebola is very different from a pandemic infectious disease. And to be honest, the R\&D Blueprint was focused on outbreak-prone viral diseases that could have big public health impacts at local levels, but that are not at the highest risk of turning into pandemics. A pandemic situation is very different.

The landscape here is much more complex. But I still believe that we have to have the WHO as a convening platform to discuss the issues, the key priorities, to define model protocols and also to discuss the current evidence for treatments. 